
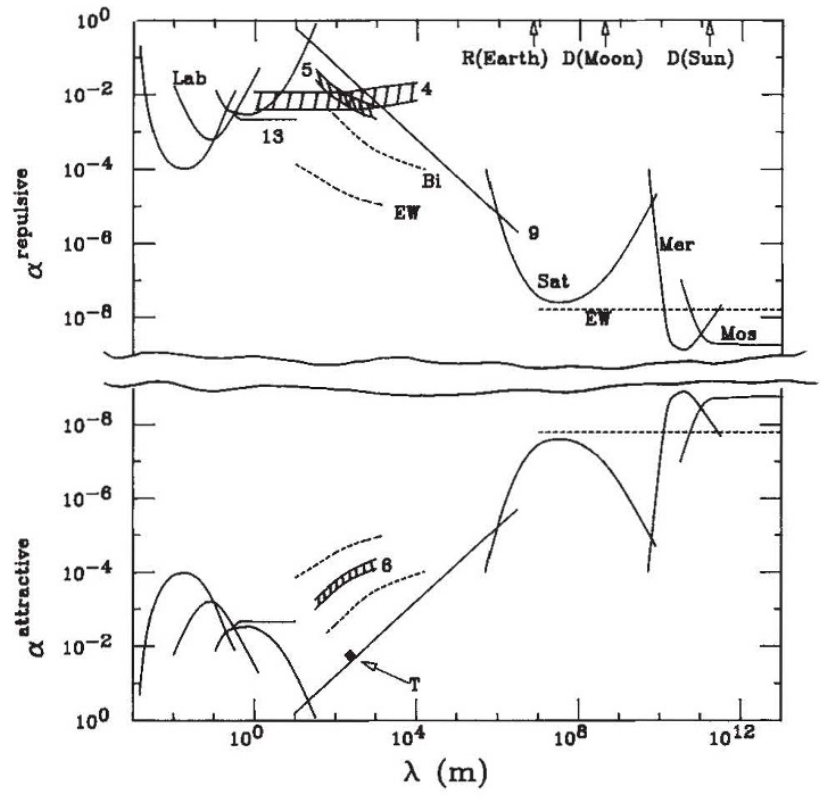

FIG. 2 Constraints on the strength (relative to G) of a Yukawa interaction coupling to $B$, for ranges $\lambda$ between $1 \mathrm{~mm}$ and $10^{13} \mathrm{~m}$. The positive results are shown as the shaded regions labelled 4 , 5 and 6 , in accord with the references. T denotes the best singleYukawa fit to the revised tower data of Eckhardt et al., who are still in the process of determining uncertainties. For clarity only the most stringent of the many null results are shown, which preclude values of $\alpha$ greater than the corresponding constraint curves. Bounds that predate the fifth force controversy (see ref. 2) arise from laboratory $1 / r^{2}$ tests (Lab), from comparisons of the orbits of manmade satellites with that of the moon (Sat), from the precession of Mercury (Mer), and from the equivalence principle experiment performed in Moscow by Braginskii and Panov (Mos). Line 9 is from the freefall experiment of Niebauer et al. ${ }^{9}$, and 13 from the Eöt-Wash torsion balance experiment ${ }^{13}$. The dashed segments EW and Bi show the results reported at Moriond by the Eöt-Wash group and by Bizzeti et al..

emptying of the lock. The data analysis sought any deflection of the balance that was correlated with water level, and none was found. Results from these isospin experiments are presented in Fig. 1.

New data were presented from two terrestrial-attractor equivalence-principle experiments. A floating-sphere experiment reminiscent of the earlier one by Thieberger ${ }^{5}$ was performed on a largescale escarpment in Italy, by P. G. Bizzeti and collaborators (Univ. Firenze). After a careful search for systematic errors, the experimenters concluded that there was no discernible difference between the hillside's action on the submerged nylon sphere and its influence on the surrounding water. The upper limit on the horizontal acceleration difference of the two materials is $2.4 \times 10^{-9}$ gal. This null result is particularly significant for $1.5 \mathrm{~km}<\lambda<16 \mathrm{~km}$.

My colleague E. Adelberger presented preliminary hillside results that our team has obtained with a much improved version of our torsion balance. We observe no acceleration difference between beryllium and aluminium at the level of $4 \times 10^{-i 1}$ gal, establishing the most stringent limit thus far on a composition- dependent interaction for $10 \mathrm{~m}<\lambda<1.5 \mathrm{~km}$. The current experimental limits on $\alpha(\lambda)$ are shown in Fig. 2, with the various results interpreted in terms of $q_{\varsigma}=B$.

The initial fifth-force hypothesis of an intermediate-range interaction with $q_{5}=B$ is not consistent with experiment. In fact no physically reasonable phenomenological model advanced to date can account for all the data, which is not too surprising given the apparent contradiction in the results.

Applying Occam's razor to the positive observations leads to the suspicion that there are unappreciated sources of systematic error in one or more of these delicate and demanding experiments. It should nevertheless be remembered that only one of the positive observations need be convincingly verified for there to be evidence of new physics. Theorists have shown that a feeble intermediate-range interaction could come about in various ways, and could be readily accommodated in several pictures of the world beyond the standard model. The possibility that these experiments may provide a window into physics that is well outside the reach of any existing or planned accelerator is sufficient motivation to continue the search.

Christopher Stubbs is in the Department of Physics, University of Washington. Seattle, Washington 98195, USA.

Fischbach E Sudarsky D Szafer A \& Talmadge, C Phys. Rev Lett. 56, 3-6 (1986)

2. De Rújula, A. Nature 323, 760-761 (1986)

3. Eckhardt, D. H., Jekeli, C., Lazarewicz, A.R., Romaides, A.J. \& Sands, R.W. Phys. Rev. Lett. 60, 2567-2570 (1988)

4tacey, F. et al. Rev. Mod. Phys. 59, 157-174 (1987)

5. Thieberger, P. Phys. Rev. Lett. 58, 1066-1069 (1987).

6. Boynton, P., Crosby, D., Ekstrom, P. \& Szumilo, A. Phys Rev. Lett. 59, 1385-1389 (1987).

. Stubbs, C.W. et al. Phys. Rev. Lett. 58, 1070-1073 (1987).

8. Fitch, V., Isaiah, M.V. \& Palmer, M.A. Phys. Rev. Lett. 60 1801-1804 (1988).

. Niebauer, T., McHugh, M.P. \& Faller, J.E. Phys. Rev. Lett 59, 609-612 (1987).

10. lacopini, E. Nature $328,578(1987)$

11. Maddox, J. Nature 329, 283 (1987)

12. Adelberger, E.G. et al. Phys. Rev. Lett. 59, 849-852 1790 (erratum) (1987)

13. Stubbs, C.W. et al. Phys. Rev. Lett. 62, 609-612 (1989)

14. Bennett, W.R. Jr. Phys. Rev. Lett. 62, 365-368 (1989).

\section{Digital puppetry}

Television transmission is absurdly inefficient. A TV picture is retransmitted in its entirety about 30 times a second, taking up about 6 megahertz of bandwidth; the new high-definition format will need 30 megahertz. Yet the incremental optical changes could probably fit comfortably into a mere few kilohertz.

So Daedalus's new Electronic Theatre TV system makes no attempt to transmit a picture. Like the human perceptual system, it codes the scene as a physical model: a 'set' or 'backdrop' with a specified appearance, in front of which a 'cast' of objects (furniture, people, cars) stand or move in a specified sequence of positions. From this description, together with defined lighting and camera position, an image-reconstruction routine in the receiver works out what the scene must look like, and displays it.

Transmission bandwidth is utterly minimized. The transmitter downloads its backdrop and cast of characters only once per scene, like a word processor downloading to a printer the font in which a document is to be printed. Thereafter 'stage directions', simple sequences of spatial coordinates and details of changes of appearance, suffice to update the image. Massive real-time computing effort is needed both at transmitter and receiver; indeed, the scheme stretches existing technology to its limits. But since computing speed and power are increasing at a hectic pace, while communication bandwidth (especially geostationary-satellite bandwidth) is ever more scarce and costly, Electronic Theatre should soon be commercially feasible.

The new system will change production methods as well as transmission. Producers and playwrights will increasingly by-pass the camera and studio altogether. They will write a show or a play directly as a script of stage directions - calling up backdrops and casts from stock digitized libraries, manoeuvring them in program form, and studying the resulting visual effect. Viewers, too, will gain. A television set will be able to substitute a local 'font' of items and characters into any broadcast: turning a global soap-opera, for example, into a national, or even a family, story. The chilling uniformity threatened by global TV will be mitigated. Mischievous viewers who want their news read by $\mathrm{Mr}$ Gorbachev in drag, say, will also have their wish. And like the live theatre itself, an Electronic Theatre production can be endlessly renewed and re-interpreted. Old dramas will be re-set in different locations, transferred to nostalgic eras, or equipped with exotic characters. Electronic Theatre will not only release bandwidth for thousands of new channels: it will provide the semblance of variety to fill them. David Jones 